\title{
New approach for the bainite start temperature calculation in steels
}

\author{
C. Garcia-Mateo ${ }^{* 1}$, T. Sourmail ${ }^{2}$, F. G. Caballero ${ }^{1}$, C. Capdevila ${ }^{1}$ and \\ C. García de Andrés ${ }^{1}$
}

The bainite start temperature $B_{\mathrm{s}}$ is defined as the highest temperature at which ferrite can transform by a displacive transformation. A common observation is that the bainite start temperature is very sensitive to the chemical composition, indicating that the influence of solutes is more than just thermodynamic. Empirical linear regression models have long been used to calculate the $B_{\mathrm{s}}$ in a limited range of compositions. This paper attempts to create an empirical model of wider applicability and higher accuracy by means of neural networks. The results are compared with those calculated using the thermodynamic theory for bainite transformation, revealing that in general this theory agrees with the experimental results, but some discrepancies can still be found when the alloys are heavily alloyed.

Keywords: Bainite start temperature, Neural network, Bayesian framework, Thermodynamics theory

\section{Introduction}

Because of its mechanical properties and low cost, bainitic steel is being increasingly used in a number of industrial applications, ranging from rails to formula one gearboxes. The bainitic structure develops at temperatures between that for pearlite formation and the martensite start temperature. The exact value of these temperatures depends strongly on the elements present in the steel, and considerable work has been devoted to developing quantitative models for their compositional dependency. Early approaches for predicting the bainite $B_{\mathrm{s}}$ and the martensite start $M_{\mathrm{s}}$ temperatures essentially consisted of the fitting of simple linear empirical relationships. ${ }^{1-9}$ Though easily communicated and interpreted, such methods often have very limited ranges of applicability because of their inability to grasp interactions or nonlinear effects. With the development of calculation frameworks such as CALPHAD, which allow prediction of thermodynamic properties of complex systems from data collected on simpler ones, more physically relevant approaches relying on the satisfaction of some thermodynamic criterion have gained importance. ${ }^{10,11}$ These are briefly introduced in the next section. In general, such models extrapolate considerably better than the early empirical approaches. However, they suffer a number of drawbacks:

(i) being essentially self-consistent, these approaches require that identical thermodynamic

1Department of Physical Metallurgy, Centro Nacional de Investigaciones Metalúrgicas (CENIM), Consejo Superior de Investigaciones Científicas (CSIC), Avda. Gregorio del Amo, 8, 28040 Madrid, Spain

${ }^{2}$ Department of Materials Science and Metallurgy, University of Cambridge, Pembroke Street, Cambridge CB2 3QZ, UK

*Corresponding author, email cgm@cenim.csic.es databases are used in the predictions as those which were used for the derivation of the criteria. With the multiplication of databases (SGTE SSOL, NPL plus, TCFE, Kmart), this is increasingly becoming a problem. Recent work by the authors ${ }^{12}$ shows, for example, that the recent improvement ${ }^{13}$ on a model developed by Ghosh and Olson ${ }^{14}$ for predicting $M_{\mathrm{s}}$ performs significantly worse than the original model if the appropriate database is not used

(ii) such approaches rely on the availability of expensive thermodynamic calculation software, the costs of which are hardly justified for such an application alone

(iii) the empirical component is not eliminated but displaced to lower levels of the model.

New empirical methods such as neural network (NN) analysis offer attractive advantages, being not only easily distributed and self-sufficient but also able to cover arbitrarily large ranges of data. As any other method, their domain of applicability is somewhat determined by the data available at the time the model is defined. However, a feature unique to the method employed in the present work is the ability of the model to accompany its predictions by an indication of their reliability.

\section{Predicting the $B_{\mathrm{s}}$ temperature}

In this section a brief description of neural network modelling is presented along with the thermodynamic theory for bainite transformation. The latter is necessary in order to understand the results and to be able to compare them with the empirical predictions obtained by means of the NN model developed in this paper. 


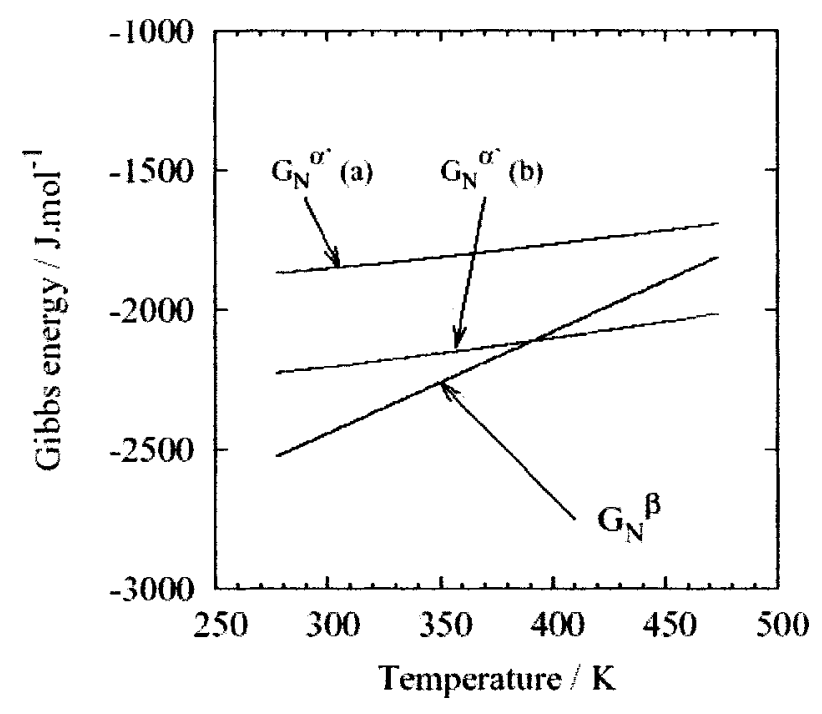

$a$ for $\mathrm{Fe}-0.32 \mathrm{C}-0.6 \mathrm{Si}-0.58 \mathrm{Mn}-0.21 \mathrm{Mo}$ (wt-\%), $b$ for $\mathrm{Fe}$ $0 \cdot 72 \mathrm{C}-1 \cdot 3 \mathrm{Si}-3 \cdot 58 \mathrm{Mn}-0.21 \mathrm{Mo}-1 \cdot 03 \mathrm{Cr}$ (wt-\%)

1 The critical driving force for martensitic type nucleation, calculated according to Ghosh and Olson $\left(G_{\mathrm{N}}^{\alpha^{\prime}}\right)$ and the function as proposed by Bhadeshia $\left(G_{N}^{\beta}\right)$

\section{Thermodynamics modelling}

For metastable austenite to undergo bainitic transformation, the driving force must be sufficient to allow nucleation and growth. Because of the displacive nature of the transformation, significant strains are generated, which can be estimated. ${ }^{15}$ For growth to occur, the driving force must exceed the strain energy. The nucleation of bainite is generally supposed to occur via formation of a small volume with bcc structure, bounded by an array or dislocations. ${ }^{11}$ In this context, the critical driving force for nucleation of bainite $\left(G_{\mathrm{N}}\right)$ is defined as that which allows for propagation of the interface by dislocation movement against the lattice friction. ${ }^{11}$ It is therefore possible to define quantitative thermodynamics criteria for the bainite transformations

$$
\begin{array}{ll}
\text { Nucleation } & \text { Growth } \\
\Delta G_{\text {nuc }}<G & \Delta G_{\text {growth }}<G_{S}
\end{array}
$$

where $\Delta G_{\text {nuc }}$ and $\Delta G_{\text {growth }}$ are the driving forces relevant to the nucleation and growth phenomena respectively; these will be defined later. In the case of bainite, it is assumed, following Bhadeshia, ${ }^{11}$ that nucleation occurs with carbon diffusion, while growth is fully displacive. It has been proposed that the stored energy $\left(G_{\mathrm{S}}\right)$ is about $400 \mathrm{~J} \mathrm{~mol}^{-1}$ in bainite. The exact criteria can therefore be expressed as

$$
\begin{array}{ll}
\text { Nucleation } & \text { Growth } \\
\Delta G^{\gamma \rightarrow \gamma^{\prime}+\alpha}<G_{N} & \Delta G^{\gamma \rightarrow \alpha}<G_{S}
\end{array}
$$

where $\Delta G^{\gamma \rightarrow \alpha}$ is the driving force for fully displacive transformation and $\Delta G^{\gamma \rightarrow \gamma^{\prime}+\alpha}$ is the driving force for the para-equilibrium transformation. This strictly should be calculated using the parallel tangent construction. However, this approach is self-consistent and will therefore lead to satisfying results as long as similar quantities are used for deriving and using the model. The principle of this method is as follows: in a first time, the transformation temperature is measured (or obtained from literature) for a number of alloys of known compositions, the relevant driving force is then calculated for the observed temperatures using existing thermodynamic databases and software (such as the SGTE solution database and MT-DATA ${ }^{16}$ ). This results in a dataset of critical driving forces for different temperatures and/or compositions. Simple equations are then fitted to represent the temperature and/or composition dependency of the critical driving force. Ghosh and Olson ${ }^{13,14}$ have used semi-empirical models to estimate the composition and temperature dependency of $G_{\mathrm{N}}$ in the context of martensite formation. The composition dependency is essentially dictated by the solid solution strengthening effect of the different additions, while the temperature dependency relates to changes in the modulus of the parent austenite. Bhadeshia and others ${ }^{17,18}$ similarly reported the temperature dependency of $G_{\mathrm{N}}$ for the bainite transformation, which approximates to

$$
G_{\mathrm{N}}=3 \cdot 5463 T-3499 \cdot 4
$$

where $T$ is the absolute temperature.

Bhadeshia suggested that, for low alloy steels, composition dependencies can be neglected and, as illustrated in Fig. 1, the work of Ghosh and Olson indeed suggests that the range of composition in which this approximation is valid is relatively limited.

It must be noted that both functions have given satisfying results in their respective domain of applications, that is to say, the function proposed by Ghosh and Olson in predicting the $M_{\mathrm{s}}$ temperature, and that proposed by Bhadeshia in predicting the $B_{\mathrm{s}}$ temperature. Further work is required to obtain a unified description for $G_{\mathrm{N}}$ and understand any remaining disagreement. In the following work, we have used Bhadeshia's criteria, together with MT-DATA and the NPL plus database (based on SGTE SSOL) to calculate the free energy changes required to estimate the $B_{\mathrm{S}}$ temperature.

As discussed above, thermodynamic models introduce some physical relevance but at the same time imply the availability of sophisticated software and databases. Their domains of validity are also difficult to assess, as errors can originate both from the thermodynamics database and from the derivation of the function fitting $G_{\mathrm{N}}$, for the $B_{\mathrm{s}}$ calculation.

\section{Neural network modelling}

The most severe inconvenience with early empirical approaches such as linear regressions resides in their limited range of applicability and their lack of flexibility. Neural networks, in the present context, essentially refers to nonlinear multiple regression tools using adaptative functions, which do not suffer these limitations. The following section will not detail the technique (see, e.g. Refs. 19-22), but presents the main features of the method. The typical structure of a neural network is presented in Fig. $2 a$.

The hidden units (the second layer in Fig. 2a) take as input a weighted sum of the inputs and return its hyperbolic tangent

$$
z_{\mathrm{j}}=\tanh \sum_{\mathrm{j}} w_{\mathrm{ji}} x_{\mathrm{i}}
$$

The third layer combines these outputs using a linear 


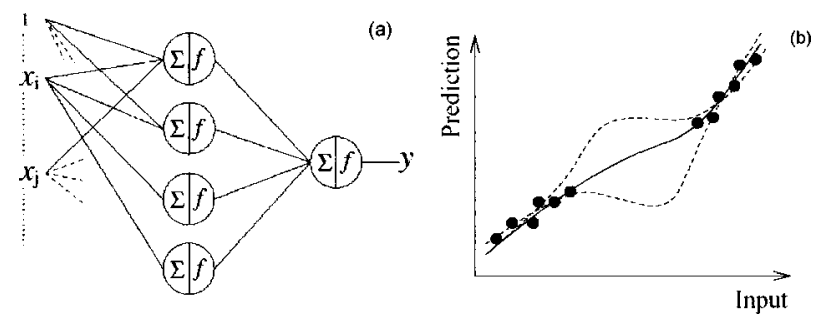

2 a The typical structure of a neural network as used for nonlinear multiple regressions. The first layer is made up by the inputs $\left(1, \ldots, x_{i}, \ldots\right)$ which are the chemical composition, the second by so called 'hidden units' and the last one is the output ( $B_{s}$ temperature). $b$ The uncertainty of fitting depends on the position in input space. Where sufficient data is available, this uncertainty is small, but where there is a lack of data, it can become large. This is important both to assess the validity of the prediction but also to identify areas where further experiments would be valuable

superposition

$$
y=\sum_{\mathrm{j}} \omega_{\mathrm{j}} z_{\mathrm{j}}
$$

where the $w_{\mathrm{ji}}$ and $\omega_{\mathrm{j}}$ are often referred to as the weights defining the network. 'Training' the network implies identifying an optimal set of weights, given some data for which the output is known. This is similar in principle to identifying the slope and intercept of the best fit line in a linear regression. As previously described the fundamental difference between this type of regression and linear regression is that neural networks correspond to adaptative functions. In traditional methods, the author fixes the form of the equation (for example, a second degree polynomial), and identifies the parameters that lead to optimal fitting of the observed data.

With neural networks however, the complexity of the function is mainly controlled by the weights themselves, so that the optimisation includes a determination of the most suitable shape for the function. This flexibility is not without a drawback: overfitting is the cause of most problems in neural network modelling. Overfitting occurs when an overly complex function is chosen, so that the noise, rather than the trend in the data, is fitted by the function. One method widely applied to limit overfitting is to perform the optimisation on only one part of the data, then use the second part to determine which level of complexity best fits the data. Recently developed approaches, which treat the problem of optimising the neural network parameters in a probabilistic manner, allow for estimation of the uncertainty of fitting. Rather than identifying optimum parameters, an optimum probability distribution of parameter values is fitted to the data. ${ }^{23}$ In regions of space where data is sparse, this distribution will be wide, indicating that a number of solutions could fit the problem with similar probabilities. If a large amount of data is available, this distribution will be narrow indicating that one shape of function is significantly more probable than any other. Because it can be quantified, the uncertainty on the determination of the network parameters can be translated into an uncertainty on the prediction error bars as illustrated in Fig. $2 b$. Further details on the method can be found in the review by Mackay. ${ }^{24}$

\section{The neural network experimental database}

A complete description of the chemical composition and the transformation temperature is required to ideally model the $B_{\mathrm{s}}$ temperature in steels. A literature survey $^{1,25-29}$ allowed us to collect 247 individual cases where detailed chemical composition and transformation temperature $\left(B_{\mathrm{s}}\right)$ were reported. Table 1 shows the list of 11 input variables used for the $B_{\mathrm{s}}$ temperature analysis. It is necessary to highlight the fact that in all the collected cases there was no interference of previous transformations or precipitation of any kind, meaning that austenite, from which bainite forms, has exactly the same chemical composition as that reported for the bulk material. Some of the alloys contain traces of elements such as $\mathrm{P}, \mathrm{S}, \mathrm{N}$ and $\mathrm{B}$, which have not been included in the model.

In relation to other existing models the range of compositions has been increased between 1-2 wt- $\%$ for $\mathrm{C}, \mathrm{Si}, \mathrm{Mn}$ and $\mathrm{V}$, and more than $5 \mathrm{wt}-\%$ in the case of $\mathrm{Cr}$, Mo and W. To the knowledge of the authors Al is included for the first time in a study of these characteristics.

\section{Results and discussion}

In order to validate the $\mathrm{NN}$ model, predicted (by $\mathrm{NN}$ and thermodynamic theory) and experimental values of $B_{\mathrm{s}}$ temperature for the database were calculated. The results are illustrated in Fig. $3 a$, the level of agreement between the experimental and those calculated $(\mathrm{NN})$ is excellent, with a square of the Pearson product moment correlation coefficient $R^{2}=0 \cdot 97$, the largest uncertanty being $\pm 35^{\circ} \mathrm{C}$. Figure $3 b$ corresponds to the results obtained on the same dataset, using the thermodynamic method as described earlier. In this case, a value of $R^{2}=$ 0.84 was obtained. In Fig. $3 b$ dashed lines representing $\pm 50^{\circ} \mathrm{C}$, help to distinguish which alloys show higher levels of discrepancy. Most of these alloys were medium/ high $\mathrm{C}$ containing $\mathrm{Cr}$ and/or Mo.

To validate the present $\mathrm{NN}$ model further, the influence of alloying elements on the $B_{\mathrm{s}}$ temperature

Table 1 Variables used to create the NN model for the calculation of the $B_{\mathrm{s}}$ temperature in steels. The table presents a summary of the range of compositions collected from literature ${ }^{1,25-29}$

\begin{tabular}{|c|c|c|c|c|c|c|c|c|c|c|c|c|}
\hline & $\mathrm{C}$ & $\mathrm{Al}$ & Si & V & $\mathrm{Cr}$ & $M n$ & Co & $\mathrm{Ni}$ & $\mathrm{Cu}$ & Mo & W & $B_{\mathrm{s}},{ }^{\circ} \mathrm{C}$ \\
\hline Min. & 0.11 & 0 & 0 & 0 & 0 & 0 & 0 & 0 & 0 & 0 & 0 & $244 \cdot 15$ \\
\hline Max. & $1 \cdot 5$ & 0.99 & $1 \cdot 67$ & $2 \cdot 1$ & $11 \cdot 5$ & $3 \cdot 76$ & 5 & $5 \cdot 04$ & 0.26 & 8 & $18 \cdot 59$ & 704 \\
\hline Mean & 0.47 & 0.004 & 0.37 & 0.058 & $1 \cdot 79$ & 0.89 & 0.06 & $1 \cdot 8$ & 0.003 & 0.75 & 0.35 & $441 \cdot 15$ \\
\hline SD & 0.18 & 0.059 & 0.31 & 0.27 & $1 \cdot 4$ & 0.48 & 0.44 & $1 \cdot 35$ & 0.021 & 0.83 & $2 \cdot 27$ & $109 \cdot 52$ \\
\hline
\end{tabular}

$\mathrm{SD}$ is standard deviation. Concentrations are in wt-\% 

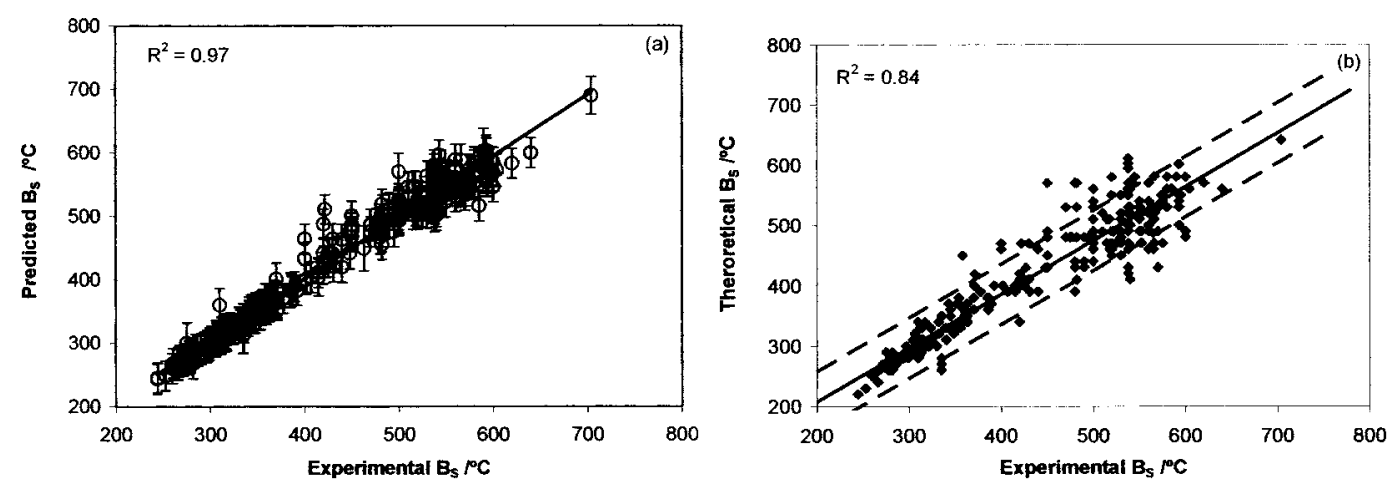

$a$ using the neural network model created; $b$ using thermodynamic theory; dashed lines represent $\pm 50^{\circ} \mathrm{C}$

3 Comparison between the calculated and measured values of $\boldsymbol{B}_{\mathrm{s}}$ temperature

has been studied for ternary systems of the type $\mathrm{Fe}-$ $0 \cdot 4 \mathrm{C}-\mathrm{X}$ and binary system $\mathrm{Fe}-\mathrm{C}$ for $\mathrm{C}$ influence (Fig. 4). It is worth emphasising the unique feature of the method employed in this paper: the ability of accompanying its predictions by an indication of their reliability. As illustrated in Fig. 2, where sufficient data is available, the uncertainty is small, and the uncertainty becomes larger where there is a lack of data, which is the case of those elements where the distance between the mean and maximum concentration is large (Table 1). The case of $\mathrm{Cr}$, Mo, $\mathrm{W}$ and $\mathrm{C}$ at high concentrations is very illustrative (Fig. 4 and Table 1). On the other hand very reasonable levels of agreement are achieved in all ranges of compositions for the other elements.

It is possible to distinguish the alloying elements according to their influence on the $B_{\mathrm{s}}$ temperature. Therefore, the general lineal trend of reducing the transformation temperature for elements as $\mathrm{C}, \mathrm{Mn}, \mathrm{Ni}$, $\mathrm{W}$ and Mo is that also observed by other authors. ${ }^{1,4,30}$ Other groups gathered elements that increase the
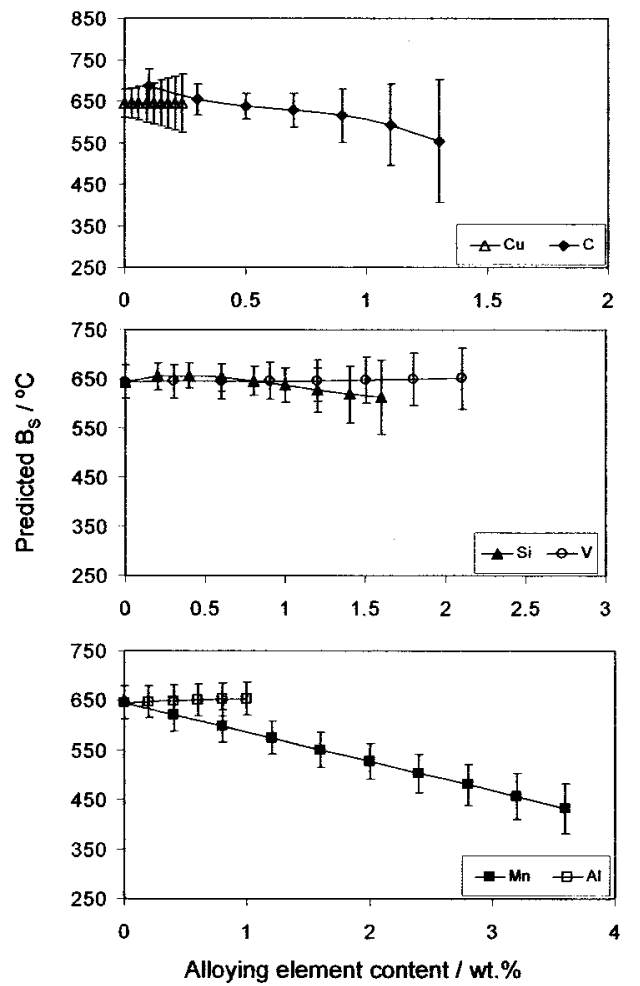

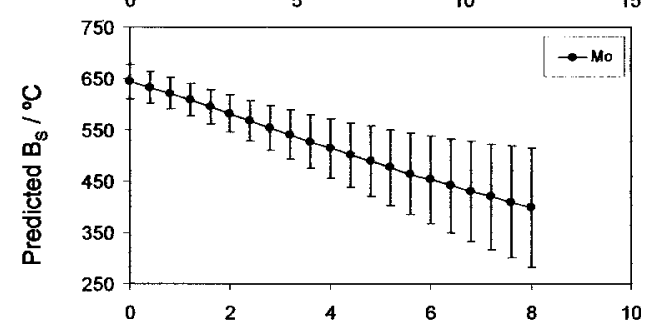

bainitic transformation temperature ( $\mathrm{Co}, \mathrm{Al}$ and $\mathrm{V})$. In this case the only reference found to the influence of $\mathrm{V}^{31}$ is opposite to that just described. There is only one element $(\mathrm{Cu})$ that has no influence on the $B_{\mathrm{s}}$ in the limited range of compositions studied.

Finally there is a group of elements that cannot be described in terms of a simple linear relationship. Up to additions of about $0.5 \mathrm{wt}-\% \mathrm{Si}$ the $B_{\mathrm{s}}$ increases; above that concentration a decrease of the temperature is predicted. This behaviour would explain that in different empirical formula used in the fitting of simple linear relationships, the influence of $\mathrm{Si}$ is either neglected or it has different signs. Another case of nonlinear behaviour is $\mathrm{Cr}$. Although the error bars become larger as the concentration increases and no accurate description of the effect can be predicted, the general trend is a reduction in $\mathrm{Cr}$ influence above concentrations of $3-$ $4 \mathrm{wt}-\%$.

In a similar scheme the latter analysis has been carried out by means of the thermodynamic model (Fig. 5). In
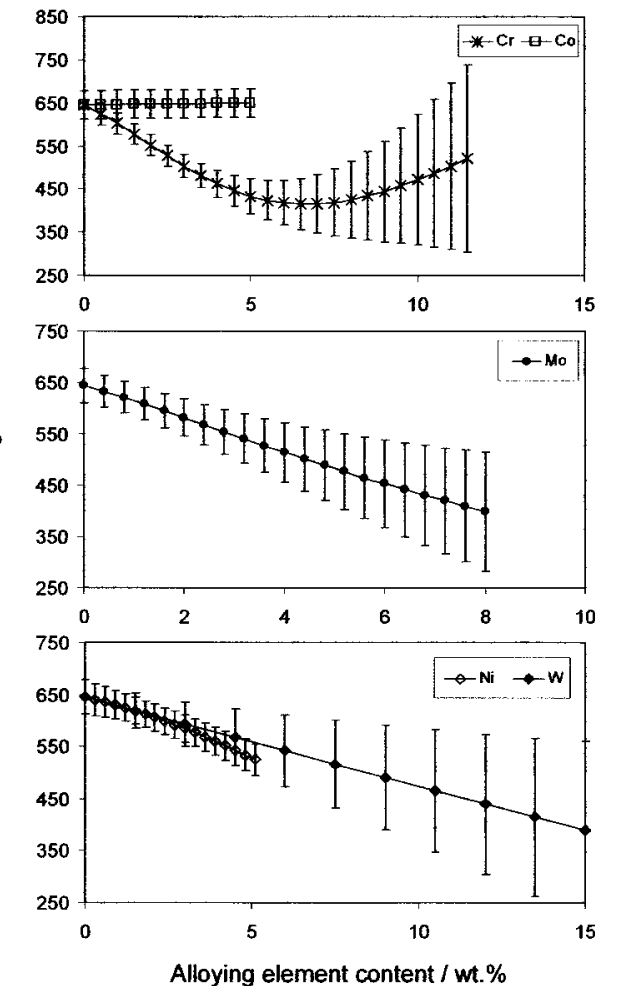

4 Effect of alloying elements on the $B_{s}$ temperature in Fe-0.4C-X steels and the binary system Fe-C for $\mathrm{C}$ influence, according to the neural network model created 

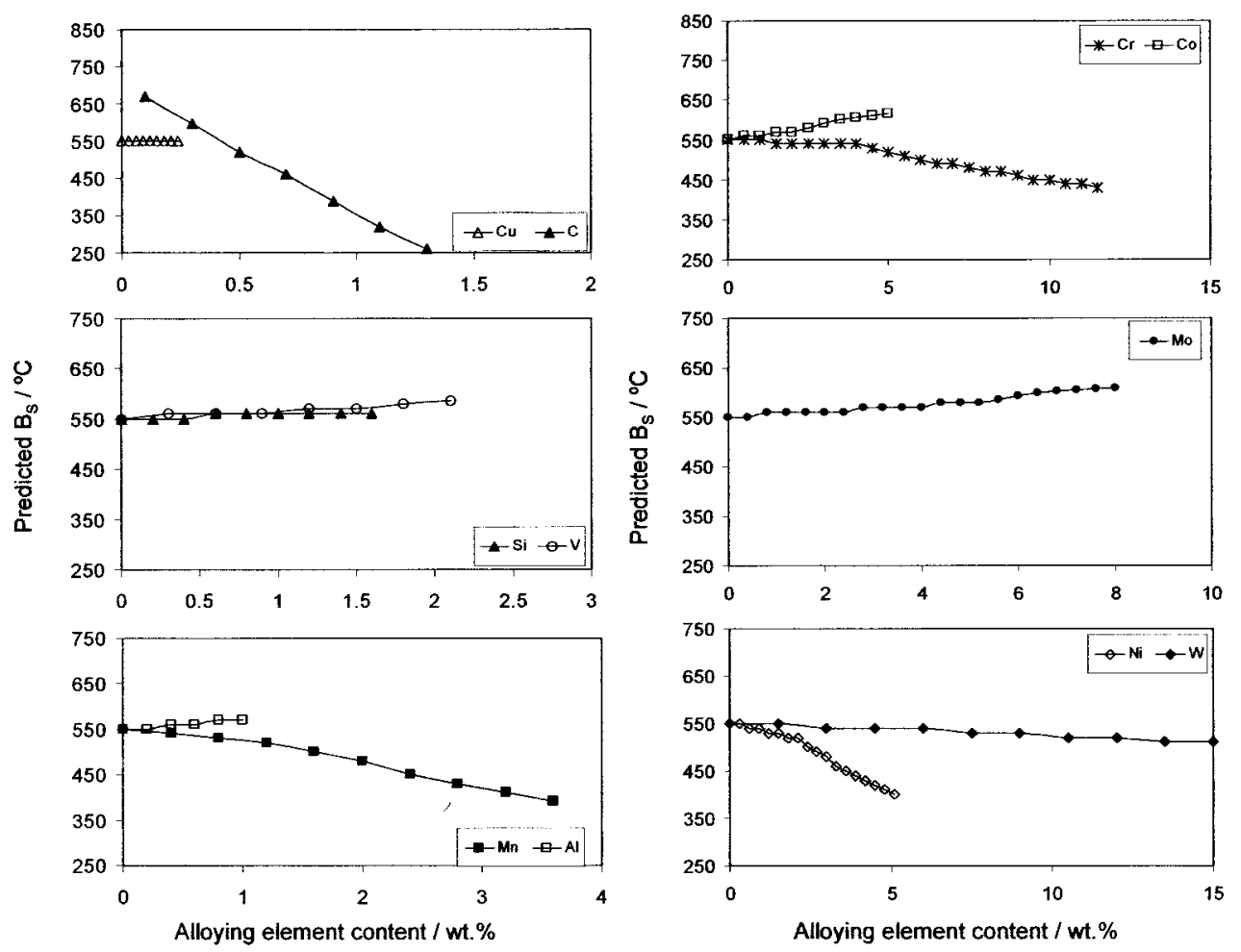

5 Effect of alloying elements on the $B_{\mathrm{s}}$ temperature in Fe-0.4C-X steels and the binary system Fe-C for $\mathrm{C}$ influence, according to Bhadeshia's thermodynamic theory for bainitic transformation

almost all elements discussed in this paper, the general trend is that predicted by the NN model. Discrepancies are found in: $\mathrm{Si}$, which according to the thermodynamic calculations does not affect the transformation temperature; $\mathrm{Cr}$, which exhibits a moderate effect up to concentrations of about $4 \%$ and then a more pronounced slope as the concentration increases. Special attention should be paid to Mo. As a ferrite stabiliser it will increase the driving force of the Gibbs energy difference, an effect that is predicted by the theory, increasing $B_{\mathrm{s}}$ with increasing Mo concentrations (Fig. 5). However, this is not the trend that is predicted by the NN model (Fig. 4). Mo reduces the $B_{\mathrm{s}}$

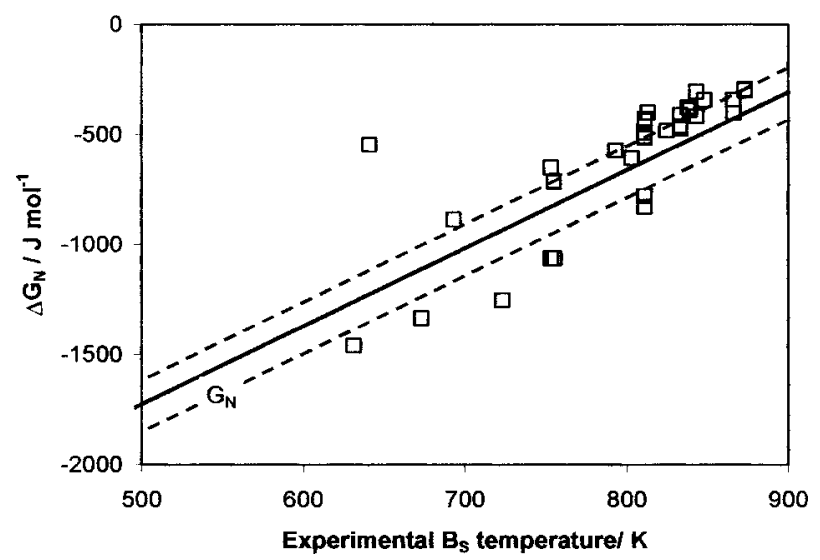

- - - - represents the limits within which are the alloys used for the calculation of $G_{N}$ in Ref. 18, $\square$ represents the free energy change for nucleation at the experimental $B_{\mathrm{s}}$ temperature for the alloys exhibiting higher levels of discrepancy in Fig. $3 b$

6 Plot of the critical driving force for nucleation $G_{N}$ temperature, emphasising that in general all the transformation temperatures calculated using thermodynamic model are underestimated if compared with those predicted by the NN model.

Focussing on the alloys that in Fig. $3 b$ exhibit higher levels of discrepancy, mainly $\mathrm{Cr}$ and/or Mo containing steels, and following the procedure described in Ref.18, the critical driving force for nucleation for these alloys was compared with $G_{\mathrm{N}}$ (equation 1). The results are presented in Fig. 6, highlighting the fact that the function $G_{\mathrm{N}}$ needs to take into account the chemical composition in order to make more accurate predictions of this critical driving force, which finally will lead to a better correlation between the experimental $B_{\mathrm{s}}$ and those calculated using the thermodynamic theory.

The NN model also has been tested against the Steven-Haynes ${ }^{1}$ and $\mathrm{Lee}^{4}$ empirical formulas. For that purpose two sets of arbitrary chemical compositions were generated, one corresponding to the range of applicability of both empirical formulas, ${ }^{1,4}$ Fig. $7 a$, and the other covering the compositions given in Table 1, Fig. 7b. As expected, in the first case the level of agreement is very good, but out of their range of applicability the empirical formulas are unable to perform, the higher discrepancy being for temperatures below $400^{\circ} \mathrm{C}$, which correspond to the more heavily alloyed steels (see Fig. $7 b$ ). In Fig. $7 c$ results using thermodynamic theory and the NN model are presented in both the above described ranges. Although a good level of agreement is achieved it is still evident that theoretical calculations predict a lower $B_{\mathrm{s}}$ temperature than the neural network model and most of the alloys close to or outside the lines of $\pm 50^{\circ} \mathrm{C}$ are those heavily alloyed or containing $\mathrm{Cr}$ and/or Mo as well as medium/ high C levels. 

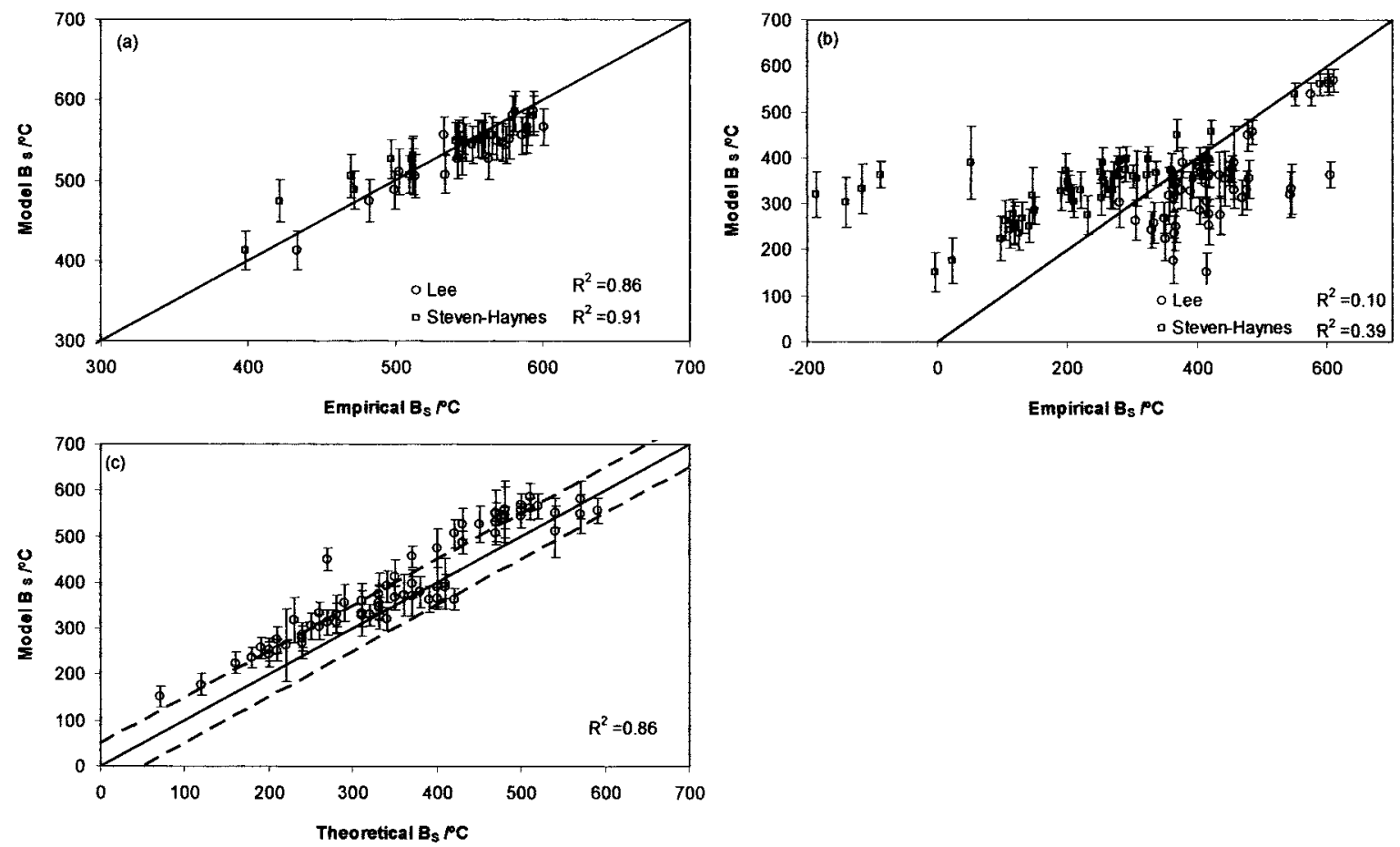

a range of applicability of these expressions; $b$ in the range given in Table $1 ; c$ comparison of the model and theory in all ranges, dashed lines represent $\pm 50^{\circ} \mathrm{C}$

7 Comparison of the NN model created in this paper against two well known empirical formulas ${ }^{1,4}$

\section{Conclusions}

It has been shown that by using the $B_{\mathrm{s}}$ neural network model described in this paper, important developments are introduced in relation with the existing empirical and thermodynamic approaches. Therefore all predictions made are accompanied by an estimation of their reliability and nonlinear relationships, such as $\mathrm{Cr}$ and $\mathrm{Si}$, which are easily identified.

Comparison with the theory has revealed some important discrepancies in terms of the degree of influence in almost all of the elements studied, and even in the trend describing their effect, which is the case of $\mathrm{Cr}$ and Mo. The discrepancies may be explained in terms of the critical driving force for nucleation $G_{\mathrm{N}}$, a function derived for low alloy steels where the effect of the chemical composition can be neglected, however for steels where the solid solution strengthening effect of different additions is relevant, steels containing $\mathrm{Cr}$ and/or Mo or heavily alloyed, thermodynamic theory predictions of the $B_{\mathrm{s}}$ temperature are not that accurate if compared with experimental or neural network model values (Fig. 4). Further work is required to introduce composition dependence on the description of the universal nucleation function $G_{\mathrm{N}}$, a key factor in the bainite start temperature determination.

\section{Acknowledgements}

The authors acknowledge the financial support from the Spanish Ministerio de Ciencia y Tecnología (projectMAT 2001-1617). F. G. Caballero would like to thank the Spanish Ministerio de Ciencia y Tecnología for the financial support in the form of a Ramón y Cajal contract (Programa RyC 2002). The authors also would like to acknowledge Professor H. K. D. H. Bhadeshia for comments and discussions during the preparation of this manuscript.

\section{References}

1. W. Steven and A. G. Haynes: J. Iron Steel Inst., 1956, 183, 349359.

2. T. Kunitake and Y. Okada, J. Iron Steel Inst. Japan, 1998, 84, 137141 .

3. J. S. Kirkaldy and D. Venugopalan: 'Phase transformations in ferrous alloys', (ed. A. R. Marder and J. I. Goldstein), 125; 1984, Warrendale, PA, TMS-AIME.

4. Y. K. Lee: J. Mater. Sci. Lett., 2002, 21, 1253-1255.

5. P. Payson and C. H. Savage: ASM Trans., 1944, 33, 261-280.

6. L. A. Carapella: Metal. Prog., 1944, 46, 108.

7. K. W. Andrews: J. Iron Steel Inst., 1965, 203, 721-726.

8. A. E. Nehrenberg: Trans. AIME, 1946, 167, 494-501.

9. R. A. Grange and H. M. Stewart: Trans. AIME, 1946, 167, 467501.

10. G. B Olson and M. Cohen: Metall. Trans. A, 1976, 7A, 18971923.

11. H. K. D. H. Bhadeshia: 'Bainite in steels', 2nd edn; 2001, London, Institute of Materials.

12. T. Sourmail and C. Garcia-Mateo: 'Critical assessment of models for predicting the $M_{\mathrm{s}}$ temperature of steels', Comp. Mater. Sci., to be published.

13. G. Ghosh and G. B. Olson: J. Phase Eq., 2001, 22, 199-207.

14. G. Ghosh and G. B. Olson: Acta. Metall. Mater., 1994, 41, 33613370 .

15. H. K. D. H. Bhadeshia: Acta Metall., 1981, 29, 1117-1130.

16. 'MT-DATA: phase diagram calculation software', National Physical Laboratory, Teddington, UK, 2003, http:// www.npl.co.uk/npl/cmmt/mtdata/.

17. A. Ali and H. K. D. H. Bhadeshia: Mater. Sci. and Tech., 1990, 6, 781-784.

18. C. Garcia-Mateo and H. K. D. H. Bhadeshia: Mater. Sci. Eng. A, 2004, 378, 289-292.

19. H. K. D. H. Bhadeshia: ISIJ Int., 1999, 39, 966-979.

20. T. Sourmail, H. K. D. H. Bhadeshia and D. J. C. MacKay: Mater. Sci. Technol., 2002, 18, 655-663.

21. C. Capdevila, C. Garcia-Mateo, F.G. Caballero and C. Garcia de Andres: Mat. Sci. Eng. A, 2004, 386, 354-361. 
22. T. Sourmail and C. Garcia-Mateo: Comp. Mater. Sci., 2005, 34, 213-218.

23. D. J. C. Mackay: Neural Computation, 1992, 4, 448-472.

24. D. J. C. Mackay: Comput. Neural. Syst. 1995, 6, 469-505.

25. H. E. Boyer: 'Atlas of isothermal transformation and cooling transformation diagrams'; 1977, Metals Park, OH, American Society for Metals

26. 'Atlas of isothermal transformation of B.S. En Steels', Special Report No. 56, 2nd edn; 1956, London, The Iron and Steel Institute.
27. L. C. Chang: Metall. Trans. A, 1999, 30, 909-916.

28. C. Garcia-Mateo, F. G. Caballero and H. K. D. H. Bhadeshia: ISIJ Int., 2003, 43, 1821-1825.

29. G. F. Vander Voort (ed.): 'Atlas of time-temperature diagrams for irons and steels', 1991; Metals Park, OH, ASM International.

30. R. L. Bodnar, T. Ohhashi and R. I. Jaffee: Metall. Trans. A, 1989, 20, $1445-1460$

31. C. L. Zhenbo Zhao, L. Yunxu and D. O. Northwood: J. Mater. Sci., 2001, 36, 5045-5056. 